\title{
The Project Design of the Tracked Vehicle Hydraulic Mechanical Differential Steering
}

\author{
Zhaozhong Yang ${ }^{1, a}$, Liwei Wang ${ }^{2, b}$, Caoyang Shi ${ }^{3, \mathrm{c}}$ \\ 1,2 troops 63981, Wuhan, 430311, China \\ ${ }^{3}$ A Agent's Room, Zhangjiakou, 075041, China

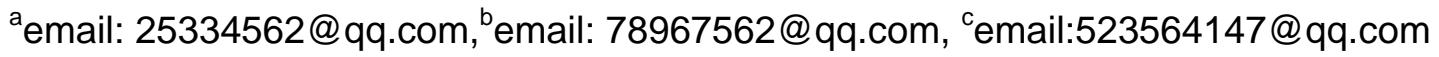

Keywords: Tracked vehicle; The hydraulic mechanical differential steering; Design

\begin{abstract}
Based on the principle of hydraulic mechanical stepless transmission is tracked vehicle hydraulic mechanical differential steering system can effectively improve the vehicle's steering can win, is has good prospects for development of a tracked vehicle steering model. Papers on tracked vehicle and its present situation and trend of development of the steering system, analysis of the hydraulic mechanical differential steering system structure, working principle, the vehicle steering lung can and on the basis of the research and application status at home and abroad, puts forward the main content of the steering system research and to solve the problem. In the output shunt transmission and input shunt two basic hydraulic mechanical transmission based on the analysis of the features, according to the requirements of the tracked vehicle steering, identified a new type of hydraulic mechanical differential steering system transmission scheme, this scheme has the advantages of simple structure and increasing the torsional speed down, caterpillar vehicle is suitable for agricultural use.
\end{abstract}

\section{Introduction}

Tracked vehicles, as a kind of "spread" the road vehicles, its unique travel system to make it a wheeled vehicle has many outstanding advantages: big traction, suitable for heavy duty operation, such as, rake and earthmoving operations; Grounding than the small, the farmland on the compaction, the extent of damage light; Across the ditch the bunds ability, etc. These advantages determine the tracked vehicle in agriculture, transportation, water conservancy, construction and is widely used in modern military field. Therefore, the research and development of the tracked vehicle level not only represents a national mechanical industry level, but also directly affects the development of the national economy.

At present, most of tracked vehicle adopts mechanical variable speed or hydraulic mechanical transmission. Have high mechanical transmission efficiency, but for the load of the adaptive ability is poor, variable speed hydraulic machinery has good adaptive ability, but its efficiency is low, how to complement each other the two has been the research focus in the field of vehicle transmission. First of all, to have a level of mechanical transmission electronic control, the shift control is one of the key technologies. Rely on hydraulic pump motors to achieve speed stepless transmission can automatically adapt to the vehicle driving resistance change, realize the stepless speed regulation, guarantee the engine work in the best working point, to improve the power performance, fuel economy, efficiency and comfort, represents the development direction of tracked vehicle transmission system.

Tracked vehicle steering system is roughly experienced a steering clutch brake, mechanical double power flow into the system and the hydraulic mechanical differential steering system, etc. For a long time, tracked vehicle steering steering clutch is often used to a brake, it has the advantages of simple structure, convenient manufacture, but also with interoperability, flexibility and steering stability, large power loss, turn to the driver and labor intensity, low speed, faults; Mechanical double power flow to the system is straight and steering both road power by mechanical transmission device, the mechanical double power flow into the system of tracked vehicle, the steering radius is still have level, the lower gears, the turning radius is smaller, still can not adapt to 
the vehicle in all the different curvature with smooth trajectory to the need of driving on the road. Application of the theory of hydraulic mechanical CVT hydraulic mechanical differential steering system can effectively improve turning performance of tracked vehicle, foreign own related products available at present, the domestic research and development is less, especially in the aspect of farm vehicles is still blank, the steering system will become the main type of tracked vehicle steering in the future.

\section{The Hydraulic Mechanical Differential Steering System}

Hydraulic mechanical differential steering is an application of the hydraulic mechanical drive, hydraulic mechanical transmission consists of hydraulic transmission, fixed axis gear drive and planetary gear transmission compound of a double power circulating dynamic type.

Hydraulic mechanical transmission system.

Hydraulic mechanical transmission is hydraulic shunt and machinery branch in parallel, achieve high efficiency through mechanical driving, by combining hydraulic drive and mechanical drive power split transmission type stepless variable speed. Power points, on the basis of the hydraulic mechanical transmission

Different can constitute the two basic transmission type, output shunt transmission type and input type.

The working process of the hydraulic mechanical transmission can be divided into mechanical working condition of single power flow hydraulic shunt (brake), hydraulic single mechanical shunt (brake) power flow conditions and working condition of hydraulic machinery double power flow three (two way to work at the same time). In addition to the working condition of mechanical single power flow, by changing the hydraulic pump capacity of shunt and mechanical shunt transmission ratio, guarantee the transmission range of output speed stepless change continuously.

\section{The hydraulic mechanical differential steering system.}

Tracked vehicle steering depends on the speed difference of implementation on both sides of the track, hydraulic mechanical transmission can realize stepless speed, hydraulic mechanical differential steering is according to the tracked vehicle steering characteristics, by using the theory of hydraulic mechanical CVT, a side track speed Rise, on the other side track speed is reduced, achieve steering of the vehicle. So hydraulic mechanical differential steering system including four indispensable constituent, is headed for variable speed system, hydraulic pressure closed loop system, reverse system and confluence planet, its, headed for variable speed system with hydraulic pressure closed loop system in parallel, can be done separately power transfer function of the vehicle. The four components of different connection type and parameters of the combination of the hydraulic mechanical differential steering system of different transmission performance, planet row of three components (round the sun, the planets and the ring gear) and hydraulic pressure closed loop system, headed for variable speed system and the system output connection type of six kinds, connection type, transmission characteristics of the system is also different. Vehicle steering, engine power points and two way transmission, power by headed for variable speed system, all the way around to a key element a. another path fixed axis gear and hydraulic pressure closed loop system, part of transfer directly to the left key element b, the other part through reverse system transfer to right key element b, two way power output after planetary line bus. Reverse system makes to planets around component b speed in the opposite direction, making produce speed difference on both sides. According to the request of tracked vehicle, if the speed of increase in side tracked in theory is equal to the other side tracked decreased, the speed of the transmission ratio of reverse system should be equal to 1 .

The working process of the hydraulic mechanical differential steering system can be divided into the single power flow straight (hydraulic self-locking) closed loop system stable steering, single power flow center (headed for variable speed system output shaft braking, steering radius is zero), single center of power flow is not stable steering (output shaft brake not headed for variable speed system, the process of vehicle steering radius on both sides of the crawler grounding external load, the influence of steering radius not sure) and double power flow to drive (two way to work at the 
same time), etc. Drivers can be adjusted by turning the steering wheel to hydraulic displacement ratio, closed loop system realize the different driving requirement.

\section{Hydraulic Mechanical Differential Steering Characteristics}

The hydraulic mechanical differential steering system as a new type of tracked vehicle steering system, compared with the traditional steering type, has the following advantages:

Excellent dynamic characteristics: the power can be proportional distribution to the crawler drive wheels on both sides, turn on both sides of the track driving wheel always transfer the power, to overcome the traditional steering system on one side of the force, but on the other side of power interruption of the defect, implementation power turning. In addition, because the system belongs to the closed circulating double power system, while the difficult road for small radius of turn can use of renewable power, thus improve the steering of the vehicle driving ability.

Stepless shift: because the system is through hydraulic stepless make produce speed difference on both sides of the track, so the vehicle can have infinite steering radius, neutral zero turning radius, greatly improved the tracked vehicle driving mobility and flexibility.

High efficiency: hydraulic mechanical differential steering system is straight and parallel transmission power, vehicle steering the steering road on both sides at the same time to attach the driving wheel of crawler equal and opposite speed, therefore, tracked vehicle average speed is not reduced, power don't interrupt, adopts the traditional steering type of steering operation with high efficiency.

Convenient operation, low labor strength: system adopts hydraulic pilot control, steering wheel, on both sides of the track driving wheel speed difference is proportional to the steering wheel travel, the driver by turning the steering wheel, control the size of the pilot valve pressure, so as to realize the vehicle's steering driving, compared with the traditional steering type of steering rod, the pilot convenient operation, low labor intensity.

Cut out good stability, turned to track precision, due to the hydraulic pressure closed loop system with hydraulic locking ability, tracked vehicle straight, to road closure, headed for stability is good. Because on both sides of the track driving wheel speed general stepless control, steering radius can implement precise control, according to the different curve road to track precision is high.

Wear light, long service life: walking system compared with the traditional steering type, tracked vehicle steering, ground track of small scuff (slip), walking system wear light, long service life.

Therefore, the hydraulic mechanical differential steering system is relatively excellent tracked vehicle steering system, but compared with the traditional steering type, hydraulic mechanical differential steering system structure is complex, high cost. With cheaper production costs for hydraulic components and people demand constantly improve the performance of tracked vehicle, the hydraulic mechanical differential steering is inevitable trend in the development of tracked vehicle.

\section{Tracked Vehicle Hydraulic Mechanical Differential Steering System Design}

The tracked vehicle hydraulic mechanical differential steering system design requirements.

Design requirements for tracked vehicle steering system, it is determined by the tracked vehicle steering performance 'requirements, basically has the following several aspects:

(1) to ensure smooth and quickly by the linear motion of tracked vehicle into along any steering radius curve movement, continuous stepless steering, neutral zero turning radius, can make the tracked vehicle with driver randomly to intend in narrow area, improve the crawler vehicles a mobility. Can make the crawler vehicles like cars, according to the smooth curve instead of the traditional mechanical steering system, turning trajectory of a similar line, improve the motion of the tracked vehicle flexibility.

(2)can make tracked vehicle steering angular velocity changes smoothly, and avoid the impact of the tracked vehicle steering angular velocity mutation caused, reduce the dynamic load, improve transmission system, steering system and walking system parts of life, improve the quality of 
tracked vehicle driving comfort and assignments.

(3)can improve the average speed of tracked vehicle. Turn the mechanical differential between the design of hydraulic system need to be able to make the crawler vehicles at little or no lower speed to safely and stability, improve the efficiency of agricultural tracked vehicle steering operation.

(4)can improve the steering performance of tracked vehicle. Tracked vehicle steering characteristics determines its steering than straight when need more power, the design of the hydraulic mechanical differential steering system need to be able to make the tracked vehicles during continuous steering will not make the engine were forced to shut down by power loss, improve the steering performance of tracked vehicle.

(5) can guarantee the stability of tracked vehicle is straight sex, should not have to turn to the trend, improve the driving stability of the tracked vehicle.

The tracked vehicle hydraulic mechanical differential steering system design.

According to the requirements of the tracked vehicle steering characteristics and design, the design of hydraulic mechanical differential steering system is mainly composed of fixed axis gear, hydraulic pressure closed loop system, headed for the convergence of variable speed system, planet, etc. Cut output variable speed system with two sets of planet gear ring gear meshing, hydraulic pressure closed loop system with a gear coupling with the sun on one side of the planet row wheel, through two pairs of gears and the other side of the planet the sun wheel connection, two rows of planets, respectively, with the final drive left/right. Engine power power flow headed for variable speed system, all the way along the power flow is controlled by a pump, motor and other components of hydraulic pressure closed loop system, two way power output by the planet carrier after confluence.

When the hydraulic pressure closed loop system does not work, the motor output speed is zero, the left and right side of the steering system planetary gear ring only input speed, because the two planets line of gear ring etc, on both sides of the track of the driving wheel speed equal, in the same direction, driving vehicles as a straight line; Output shaft brake when headed for variable speed system, right or left planet only sun wheel input speed, because the two planets of the sun between wheel and hydraulic pressure closed loop systems are a pair of gears, on both sides of the track of the driving wheel speed equal and opposite, vehicle for stable steering driving (steering radius is zero), it is especially suitable for agricultural crawler tractor suspension farm work that need to be flexible steering condition; When headed for variable speed system output shaft idling don't brake, the vehicle will be unstable center according to the situation of ground resistance to driving (steering radius is not zero); When two road work at the same time, for from the least to radius to any vehicle steering radius of left and right steering movement, which can realize stepless steering.

\section{Tracked Vehicle Hydraulic Mechanical Differential Steering System Design}

Tracked vehicle hydraulic mechanical differential steering system is to produce to instruction and performed by the actuator of a fluid control system, is the realization of tracked vehicle differential steering hydraulic machinery of key component.

The tracked vehicle hydraulic mechanical differential steering system design requirements.

According to the actual working condition of the tracked vehicle, the hydraulic mechanical differential steering system shall meet the following requirements:

(1) of the tracked vehicle steering is about two-way, steering control system should have two-way job characteristics, tracked vehicle turn left, the response of the system output speed.

(2) the hydraulic mechanical differential steering system consists of steering wheel and the wheel in the limit position, the minimum turn radius of the vehicle shall meet the requirements of each block of minimum steering radius, and can realize zero steering radius at the center of the steering. The wheel travel increases, accordingly, the turning radius is proportional to the decreased; Change direction, and the manipulation should be smooth, continuous transition.

(3) to perform system should be able to generate enough to drive moment, can overcome the vehicle steering the steering resistance moment in the process of driving. 
(4) a car travels in a straight line, steering control system, power loss and can keep good straight trajectory accuracy, stability and steering system must have certain ability of hydraulic lock.

(5) tracked vehicle reverse turn, need to be able to "U" shaped like wheeled vehicles did turned.

(6) system working stability, high reliability.

\section{The tracked vehicle hydraulic mechanical differential steering control system principle and composition.}

Differential steering system for hydraulic machinery control is, in fact, by changing the hydraulic motor output speed closed loop system, make the produce speed difference on both sides of the track, so as to realize the tracked vehicle steering. Hydraulic pressure closed loop system is hydraulic transmission system, hydraulic drive system of speed adjustment ways mainly have the throttling control of motor speed and volume control in two ways, large power consumption, low efficiency because of throttling speed control, hydraulic pressure closed loop system using volumetric speed control mode, therefore, there are three kinds of commonly used control way is mainly: variable pump and quantitative motor circuits, quantitative pump and variable motor circuit and the above two ways of combination of variable pump and variable motor circuit.

Variable pump motor circuit by changing the variable pump certain amount of displacement to change quantitative motor output speed, with large speed range, can realize stepless speed regulation in a row. When change the direction of oil pump, the motor can smooth the reversing, meet the needs of the vehicle turn left. When the load changed little, the output of the motor rotation speed and pressure change is not big, has the characteristics of constant torque speed regulation, and is suitable for the occasion of load torque change little. Tracked vehicles during steering, the steering resistance to change is not big, so the hydraulic pressure closed loop system adopts variable pump motor circuit of certain amount.

\section{Steering process analysis.}

Left when the steering wheel, hydraulic press down on the left side of the proportional pressure reducing valve, pilot valve by filling the oil pump of hydraulic oil through the proportional pressure reducing valve superior by the electromagnetic directional valve into the axial plunger variable pump, the axial plunger in the displacement of variable pump control valve to the right place, filling the hydraulic oil pump output driving axial piston swash plate of variable pump, increase the axial piston variable displacement pump, the flow of hydraulic pressure closed loop system increases, the axial plunger quantitative increase the output of the motor speed, which tracked vehicle on the right track speed increase, accordingly reduce the speed of the left side of the tracks, turn left tracked vehicle. Turn right on the other hand, when the steering wheel, hydraulic pilot valve pressure proportional pressure reducing valve, on the right side of the axial piston pump reverse variables, so that the tracked vehicle on the left side of the track speed increase, accordingly reduce the right track, the speed of the tracked vehicle shift to the right. Under the steering wheel rotation Angle, the greater the proportional pressure reducing valve, the more into the axial plunger pump variable servo cylinder pressure, the greater the axial piston swash plate Angle of variable pump, the greater the axial piston variable displacement pump, the greater the main lines of the flow rate, the greater the making of axial piston motor quantitative output rotational speed is higher, on both sides of tracked vehicle track the speed of increase and decrease respectively, the greater the speed difference on both sides of the track, the greater the tracked vehicle steering radius under the same block is smaller. When turn the steering wheel to the limit position, the greater the output flow of axial piston variable pump, the minimum turning radius of tracked vehicle, neutral when the steering radius is zero.

\section{Conclusion}

By means of hydraulic mechanical transmission type of differential steering system and feature analysis, and according to the tracked vehicle steering characteristics and design requirements, design the hydraulic mechanical differential steering system of a tracked vehicle. Analysis shows that the steering system can well meet the linear, center of tracked vehicle steering and general in typical working condition, such as driving, by comparison with advanced power steering system, 
the steering system has simple structure, the advantages of slow increase torsion, caterpillar vehicle is suitable for agricultural use. Based on tracked vehicle hydraulic mechanical differential steering system design requirements, determine the principle of hydraulic mechanical differential steering system, and select the corresponding hydraulic components. Through the steering process analysis, the system can guarantee the tracked vehicle steering performance of the process of implementation.

\section{References}

[1] Fendt.Fendt 400 Vario Technical Speeifieations, Available at:www. Fendt.com[OL], Accessed 17-11-2002.

[2] Sergio M, Francesco L .Control System Design on a Power-Split CVT for High-power Agricultural Tractors [J]. ASME TRANSACTIONS ON MECHATRONICS, 2004, Vol,. 9(3):569-579. 